\title{
A 44-Year-Old Female With Dysphagia Presenting a Thin Muscle Thickness on High-Frequency Intraluminal Ultrasound
}

\author{
Jeong Hwan Kim \\ Department of Internal Medicine, Digestive Disease Center, Konkuk University Medical Center, Seoul, Korea
}

A 44-year-old female visited the Digestive Disease Center for the evaluation of dysphagia. She complained of severe dysphagia to both solids and liquids for 5 years. Additionally, she regurgitated for 3 years and lost $10 \mathrm{~kg}$ for a year. On upper endoscopic

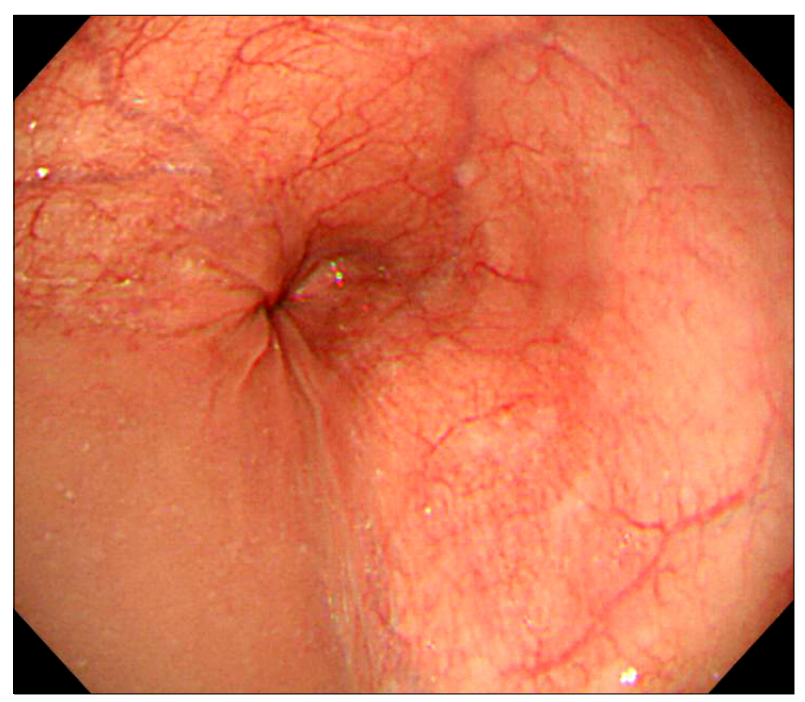

Figure 1. Upper endoscopy. Retained fluid is found in the distal esophagus and powerful contractions are observed on the esophagogastric junction. finding, the esophageal mucosa appeared to be normal, but there was retained fluid in the distal esophagus and severe contractions leading to resistance in advancing the scope to esophagogastric junction (Fig. 1). Esophagogram showed the dilated aperistaltic

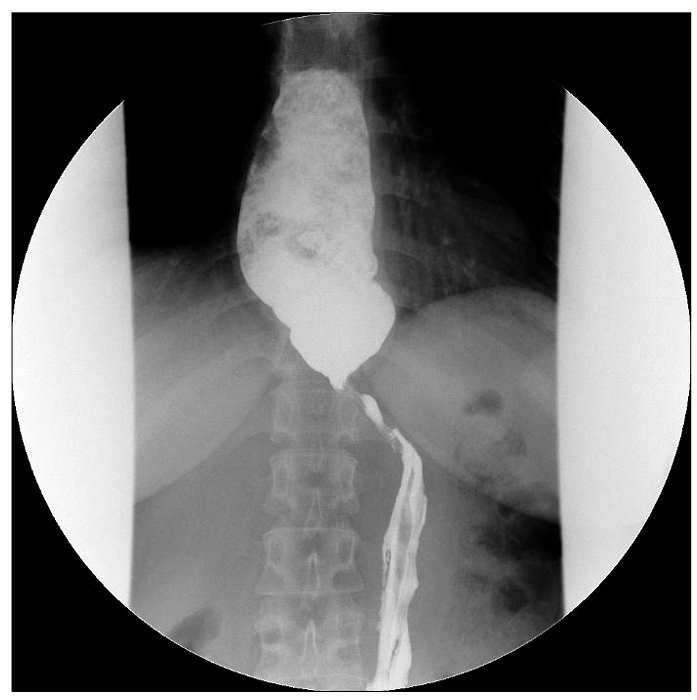

Figure 2. Esophagogram. This view shows the typical findings of achalasia: dilated aperistaltic esophagus and beaklike narrowing near the esophagogastric junction.

Received: March 27, 2011 Revised: April 7, 2011 Accepted: April 8, 2011

(c) This is an Open Access article distributed under the terms of the Creative Commons Attribution Non-Commercial License (http://creativecommons. org/licenses/by-nc/3.0) which permits unrestricted non-commercial use, distribution, and reproduction in any medium, provided the original work is properly cited.

*Correspondence: Jeong Hwan Kim, MD

Department of Internal Medicine, Digestive Disease Center, Konkuk University Medical Center, 4-12 Hwayang-dong, Gwangjin-gu, Seoul 143-729, Korea

Tel: +82-2-2030-5010, Fax: +82-2-2030-5029, E-mail: sefamily@kuh.ac.kr

Financial support: None.

Conflicts of interest: None. 


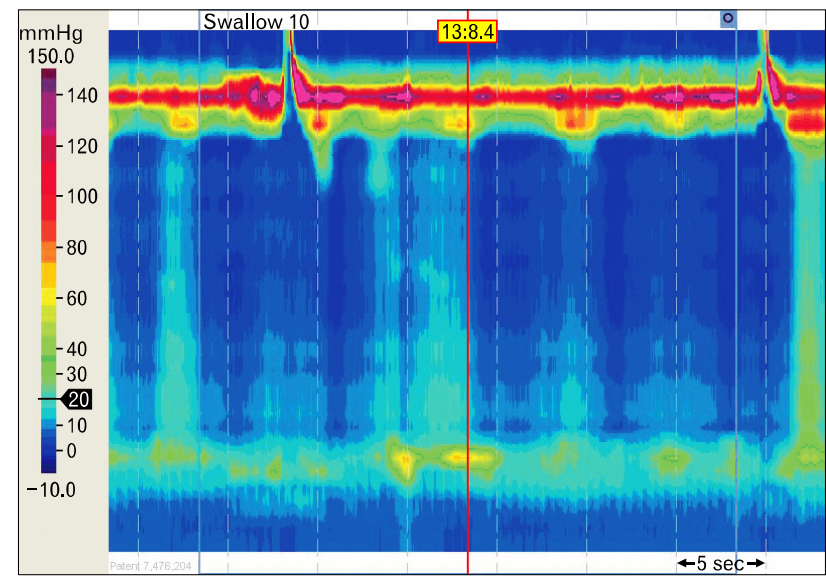

Figure 3. High-resolution manometry. There is no significant pressurization within the esophageal body with impaired lower esophageal sphincter relaxation.

esophagus and beaklike narrowing near esophagogastric junction (Fig. 2). High-resolution manometry showed no significant pressurization within the esophageal body with impaired lower esophageal sphincter relaxation (Fig. 3). These findings led to the diagnosis of type 1 achalasia based on a recent high-resolution manometry classification proposed by Pandolfino et al. ${ }^{1}$

A number of investigators have reported the use of high-frequency intraluminal ultrasound (HFIUS) to measure muscle thickness and cross sectional area (CSA) of esophagus. ${ }^{2-5}$ According to the reports using HFIUS, the primary esophageal motility disorders including achalasia, diffuse esophageal spasm and nutcracker esophagus are associated with hypertrophy of the circular as well as longitudinal muscle layers. ${ }^{4}$ However, HFIUS images in this patient showed a markedly dilated esophagus with a thin muscle thickness (Fig. 4). Additionally, even though the muscle was thin, muscle CSA was larger than the controls from our previous report. ${ }^{5}$

Interestingly, the presented case showed the thin muscle thickness and large muscle CSA on HFIUS which was different from the traditional type of achalasia presenting a thick muscle

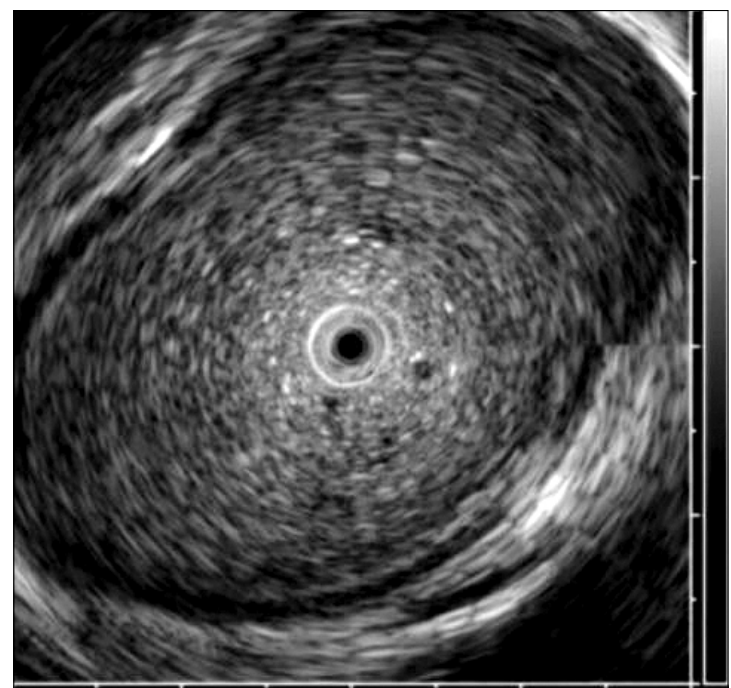

Figure 4. Muscle thickness and muscle cross sectional area (CSA) in high-frequency intraluminal ultrasound images. The image shows a markedly dilated esophagus with a thin muscle. Note that the muscle CSA is larger $\left(157 \mathrm{~mm}^{2}\right)$, even though the muscle is thin $(1.65 \mathrm{~mm})$.

thickness.

\section{References}

1. Pandolfino JE, Kwiatek MA, Nealis T, Bulsiewicz W, Post J, Kahrilas PJ. Achalasia: a new clinically relevant classification by high-resolution manometry. Gastroenterology 2008;135:1526-1533.

2. Rhee PL, Liu J, Puckett JL, Mittal RK. Measuring esophageal distension by high-frequency intraluminal ultrasound probe. Am J Physiol Gastrointest Liver Physiol 2002;283:G886-G892.

3. Takeda T, Kassab G, Liu J, Puckett JL, Mittal RR, Mittal RK. A novel ultrasound technique to study the biomechanics of the human esophagus in vivo. Am J Physiol Gastrointest Liver Physiol 2002; 282:G785-G793.

4. Dogan I, Puckett JL, Padda BS, Mittal RK. Prevalence of increased esophageal muscle thickness in patients with esophageal symptoms. Am J Gastroenterol 2007;102:137-145.

5. Kim JH, Rhee PL, Son HJ, Song KJ, Kim JJ, Rhee JC. Is all ineffective esophageal motility the same?: A clinical and high-frequency intraluminal US study. Gastrointest Endosc 2008;68:422-431. 